\title{
Epidemiology and prognostic nomogram for esophageal cancer with liver metastasis based on the Surveillance, Epidemiology, and End Results database
}

\section{Huacai Xiong}

Ningbo Medical Center Lihuili Hospital

Danfei Hu

Ningbo Medical Center Lihuili Hospital

Mingyao Li

Ningbo Medical Center Lihuili Hospital

Tian Chen

Ningbo Medical Center Lihuili Hospital

Wei Jiang

Ningbo Medical Center Lihuili Hospital

Zhenfei Xiang ( $\nabla$ xiangzhenfei1981@163.com )

Ningbo Medical Center Lihuili Hospital

\section{Research Article}

Keywords: esophageal cancer, liver metastases, database analysis, prognosis, nomogram

Posted Date: March 22nd, 2022

DOI: https://doi.org/10.21203/rs.3.rs-1283952/v2

License: (c) (i) This work is licensed under a Creative Commons Attribution 4.0 International License.

Read Full License 


\section{Abstract \\ Background}

The prognosis of esophageal cancer with liver metastasis (ECLM) is poor. This study evaluated the incidence and prognostic factors of ECLM at initial diagnosis and developed a visual nomogram to predict outcomes.

\section{Methods}

1892 eligible ECLM patients diagnosed between 2010 and 2016 were extracted from the Surveillance, Epidemiology, and End Results database (SEER). The incidence, patient overall survival (OS), and prognosis of liver metastasis were evaluated according to patient information, tumor characteristics, and therapy. Cox regression analyses were applied to evaluate prognostic factors. Based on the significant demographic and clinicopathologic factors, a novel visual nomogram was established to predict 6-month and 1-year survival for ECLM patients.

\section{Results}

The incidence of ECLM increased with age, but survival rates showed the opposite trend. From 2010 to 2016 , as age increased, the incidence also increased annually, peaking at age 70 to 79 years and then decreasing. The 1-, 3-, 6-, 9- and 12-month relative cancer survival rates for ECLM patients were 84.6\%, $61.3 \%, 44.0 \%, 31.4 \%$, and $21.8 \%$, respectively. Multivariate cox regression analysis revealed that age, gender, grade, histological type, bone metastasis, lung metastasis, brain metastasis, chemotherapy and local treatment were independent prognostic factors related to OS. Moreover, our nomogram predicted the 6-month and 1-year survival times of the population, with a C-index of 0.735 (95\% $\mathrm{Cl}$ : $0.723-0.748)$. The nomogram demonstrated good clinical applicability.

\section{Conclusions}

The incidence of ECLM increased with age, but survival rates showed the opposite trend. Age, gender, tumor characteristics, and therapy should be considered when predicting the prognosis of ECLM patients. The nomogram may improve clinicians' abilities to predict individual survival and recommend treatments for ECLM patients.

\section{Introduction}

Esophageal cancer (EC) is a malignant tumor that represents the sixth leading cause of cancer-related deaths worldwide. There were 473,000 (95\% uncertainty interval [95\% UI] 459,000-485,000) new cases of EC and $436,000(425,000-448,000)$ deaths due to EC in $2017^{[1]}$. The incidence and mortality of EC vary 
geographically, with a high burden in East Asia and Eastern/Southern Africa, where esophageal squamous cell carcinoma predominates over adenocarcinoma ${ }^{[2]}$. However, esophageal adenocarcinoma (EAC) has become the most common esophageal malignancy in Western countries ${ }^{[2,3]}$. The most common sites of distant metastasis (DM) at initial diagnosis are the non-regional lymph nodes, liver, lung, bone, and adrenal gland ${ }^{[4]}$. The prognosis of metastatic EC is poor, and the five-year survival rate is less than $5 \%{ }^{[5]}$. The liver is one of the most common sites of metastases, occurring in $35-40 \%$ of patients at the time of EC diagnosis ${ }^{[6]}$. Chemotherapy is the standard treatment for these patients, however, survival is not satisfactory ${ }^{[7,8]}$. Small trials have reported that patients with a low hepatic tumor burden have a favorable prognosis after liver resection or another local therapy ${ }^{[9,10]}$.The optimal management of patients with liver metastases is still a question of debate.

Although there have been some studies on esophageal cancer with liver metastasis (ECLM) ${ }^{[2,3,11]}$, data on the epidemiology and signatures of this disease are still unclear. To comprehensively demonstrate the epidemiological characteristics and analyze the possible prognostic factors in the survival of patients with ECLM and predict the survival time of individual patients, the Surveillance, Epidemiology and End Results (SEER) database was used in the present study. Profound knowledge of the epidemiological features and clinical characteristics of ECLM is crucial to improve patient treatment. The SEER database provides an opportunity to investigate the epidemiological characteristics and analyze the possible prognostic factors in the survival of patients with ECLM and predict the survival time of individual patients.

\section{Methods}

This retrospective study was based on data obtained from the SEER database 18 registry, which is maintained by the National Cancer Institute of the United States. We extracted raw data through online access using SEER ${ }^{\star}$ Stat software version 8.3.6 ${ }^{[12]}$. Analysis of the raw data from the SEER program was exempt from medical ethics review, and no informed consent was required. All procedures performed in studies involving human participants were in accordance with the 1964 Helsinki Declaration.

\section{Study population selection}

We limited the data to the following selection criteria: EC patients of all ages diagnosed with liver metastasis between 2010 and 2016. The patients with ECLM were selected for further research. Patients diagnosed with other tumors were exempt from the selected population. EC patients with brain, bone and lung metastases were also selected in the same manner for comparison to those with liver metastases. Patients who were diagnosed via autopsy or a death certificate or whose detailed information was unknown or blank were excluded.

\section{Data elements}


The detailed demographic and clinicopathological features for each patient are shown in Table 1. Demographic variables included age at diagnosis, gender, race, vital status and survival (in months). Cancer characteristics included the primary tumor location, grade, T stage, $\mathrm{N}$ stage, histologic type, and sites of DM. Treatment information, including chemotherapy and local treatment (surgery and/or radiation), was also collected from the database. Local treatment was defined as surgery at the primary lesion and/or radiotherapy. Histological types were based on the International Classification of Diseases Codes for Oncology (ICD-0) (adenocarcinoma: 8140, 8144, 8210, 8211, 8244, 8255, 8260-8263, 8480, 8481,8490 , and 8560 , squamous carcinoma: $8052,8070-8074,8083$, and 8094 , and others: 8012,8013 , $8033,8041,8045,8246,8574$, and 8936). Tumor stage and lymph node stage were stratified according to the 7th edition of the TNM staging guidelines released by the American Joint Committee on Cancer (AJCC). Patients with missing values or unspecified conditions on these items were excluded. Patients diagnosed by a death certificate or autopsy were also excluded. All the raw data were downloaded from the SEER website (https://seer.cancer.gov/data/) via SEER*Stat software.

Table 1 Characteristics of patients with ECLM 


\begin{tabular}{|c|c|c|}
\hline \multirow[t]{2}{*}{ Characteristic } & \multicolumn{2}{|c|}{ Total $(n=1892)$} \\
\hline & $\mathrm{N}$ & Percent (\%) \\
\hline \multicolumn{3}{|l|}{ Gender } \\
\hline Male & 1631 & 86.2 \\
\hline Female & 261 & 13.8 \\
\hline \multicolumn{3}{|l|}{ Race } \\
\hline White & 1658 & 87.6 \\
\hline Black & 148 & 7.8 \\
\hline Other & 86 & 4.5 \\
\hline \multicolumn{3}{|l|}{ Age } \\
\hline$<57$ years & 540 & 28.5 \\
\hline $58-77$ years & 1122 & 59.3 \\
\hline$\geq 78$ years & 230 & 12.2 \\
\hline \multicolumn{3}{|l|}{ Pathological grade } \\
\hline Grades 1-2 & 687 & 36.3 \\
\hline Grades 3-4 & 1205 & 63.7 \\
\hline \multicolumn{3}{|l|}{ Histological type } \\
\hline Adenocarcinoma & 1513 & 80.0 \\
\hline Squamous & 320 & 16.9 \\
\hline Other & 59 & 3.1 \\
\hline \multicolumn{3}{|l|}{ Primary tumor site } \\
\hline Lower & 1540 & 81.4 \\
\hline Upper+Middle & 244 & 12.9 \\
\hline Overlapping lesion & 108 & 5.7 \\
\hline \multicolumn{3}{|l|}{ T stage } \\
\hline $\mathrm{T} 1+\mathrm{T} 2$ & 525 & 27.7 \\
\hline $\mathrm{T} 3+\mathrm{T} 4$ & 658 & 34.8 \\
\hline Tx & 709 & 37.5 \\
\hline $\mathrm{N}$ stage & & \\
\hline
\end{tabular}




\begin{tabular}{|lll|}
\hline $\mathrm{N} 0$ & 528 & 27.9 \\
\hline Site of distant metastases & 1364 & 72.1 \\
\hline Bone & 384 & 20.3 \\
\hline Lung & 554 & 29.3 \\
\hline Brain & 82 & 4.3 \\
\hline Chemotherapy & & \\
\hline No & 670 & 35.4 \\
\hline Yes & 1222 & 64.6 \\
\hline Surgery & & \\
\hline No & 1862 & 98.4 \\
\hline Yes & 39 & 1.6 \\
\hline Radiation & & \\
\hline No & 1260 & 66.6 \\
\hline Yes & 632 & 33.4 \\
\hline Local treatment & & \\
\hline No & 1243 & 65.7 \\
\hline Yes & 649 & 34.3 \\
\hline
\end{tabular}

Abbreviation: ECLM, esophageal cancer with liver metastasis, $\mathrm{T}$, tumor, $\mathrm{N}$, lymph node, local treatment, surgery or radiation.

\section{Statistical analysis}

Patients were divided into three groups based on age by using the X-tile program (Yale University, New Haven, CT, USA) ${ }^{[13]}$. The optimal cutoff values were 57 and 77 years. For convenience, we divided the patients into 3 age groups $(0-57,58-77$, and $78+$ years $)$ for analysis. Kaplan-Meier analysis and log-rank tests were used to compare OS.

We examined the incidence for patients diagnosed between 2010 and 2016 through SEER ${ }^{\star S t a t}$ statistical software for Windows. We also compared the incidence according to the sites of DM (liver, brain, bone, and lung). The 1-, 3-, 6-, 9- and 12-month relative cancer survival rates for ECLM patients were also examined according to the sites of DM. Detailed differences between different age subgroups were also explored. Moreover, survival curves were described by using Kaplan-Meier methods after adjusting for 
age, gender, race, histological type, primary site, tumor stage, lymph node stage, sites of DM, chemotherapy and local treatment. Differences between the OS curves were compared using the log-rank test. In all statistical analyses, a $p$ value $<0.05$ was considered significant.

\section{Prognostic nomogram for OS}

Cox proportional hazards regression was used to analyze the associations between OS and relevant prognostic factors, including age, gender, race, primary tumor site, histological type, grade, tumor stage, lymph node stage, bone metastasis, lung metastasis, brain metastasis, local treatment and chemotherapy, along with treatment information, based on the SEER database. A visual nomogram based on possible prognostic factors associated with OS was established using $\mathrm{R}$ software on the basis of the Cox regression results. The discriminative ability of the prognostic model was evaluated with Harrell's concordance index (C-index). The C-index estimates the probability of concordance between predicted OS and observed OS. Calibration was performed by comparing predicted OS with observed OS against the nomogram's 6-month and 1-year predicted OS outcomes. Decision curve analysis (DCA) was also employed to evaluate the clinical usefulness of the model. All statistical analyses were completed with SPSS software (version 23, IBM Corp., USA) and R software (version 3.3.0, http://www.r-project.org/).

\section{Results}

\section{Characteristics of the study population}

4076 patients were diagnosed with ECLM between 2010 and 2016 in the United States and included in our initial analysis. After excluding patients with missing follow-up or unknown data, a total of 1892 patients were included in the present study. The demographic data of the patients and the characteristics of the tumors are shown in Table 1. According to age at diagnosis, patients were divided into three groups: $0-57$ years $(540,28.50 \%), 58-77$ years $(1122,59.30 \%)$, and 78 years or older $(230,12.2 \%)$. There was a male predominance [1631 (86.20\%) males versus 261 (13.80\%) females]. Of those who met the eligibility criteria, 1658 (87.60\%) were White, 148 (7.80\%) were Black, and 86 (4.50\%) were from another race. The primary tumor site was predominantly the lower third of the esophagus $(1540,81.40 \%)$. There was a significantly high proportion of poorly differentiated tumors $(63.70 \%)$, well differentiated tumors accounted for $36.30 \%$. The distribution of site-specific metastasis varied in the different groups. A total of $384(20.30 \%)$ patients had bone metastasis, 554 (29.30\%) patients had lung metastasis, and 82 $(4.30 \%)$ patients had brain metastasis. A small proportion of patients received primary surgery (39, $1.60 \%)$, and $1862(98.4 \%)$ patients did not undergo primary surgery. A total of $632(33.40 \%)$ patients received radiotherapy, and 1260 (66.60\%) did not receive radiotherapy or their radiotherapy status was unknown. In total, 649 (34.30\%) patients received local treatment, and the remaining $1243(65.70 \%)$ patients did not receive local treatment. A total of $1222(64.60 \%)$ patients received chemotherapy, and the remaining $670(35.40 \%)$ patients did not receive chemotherapy or their chemotherapy status was unknown. 


\section{Incidence}

The incidence of ECLM increased with age (Figure 1A), but survival rates showed the opposite trend (Figure 1D). It is notable that the survival rate of adults continued to decrease with increasing age. We then analyzed the annual incidence of patients in different age groups from 2010 to 2016 . As age increased, the incidence increased annually, peaking at age 70 to 79 years and then decreasing. We then compared the incidence of four common metastatic sites of EC (bone, brain, liver, and lung) from 2010 to 2016 and found that the most common site of metastasis was the liver (Figure 1B), followed by the lung, bone and brain.

\section{Survival analysis}

The OS of patients with EC with/without metastasis is shown in Figure 1C. All patients with metastases at the four most common sites had a worse prognosis than those without metastasis, EC patients with bone metastasis having the worst prognosis. Liver metastases were associated with better OS than bone metastases. The survival rate of patients with ECLM is shown in Figure 1C. The 1-, 3-, 6-, 9- and 12-month relative cancer survival rates for ECLM patients were $84.6 \%, 61.3 \%, 44.0 \%, 31.4 \%$, and $21.8 \%$, respectively. Kaplan-Meier survival curves also confirmed the effect of prognostic factors, including age, gender, race, histological type, primary site, tumor stage, lymph node stage, grade, site of metastasis and treatment information. The log-rank tests for all Kaplan-Meier survival curves except for those on gender $(p>0.05)$ and race $(p>0.05)$ were statistically significant $(p<0.05)$, but the curves were crossed (Figure 2$)$. We also found that the median survival time was similar and not optimistic.

\section{Prognostic factors of OS}

The prognostic factors of OS were calculated with univariate and multivariate Cox proportional hazards regression analyses (Table 2$)$. The univariate analysis indicated that age $(p \leq 0.001)$, grade $(p \leq 0.001)$, primary tumor site $(p=0.002)$, histological type $(p \leq 0.001)$, T stage $(p=0.004), \mathrm{N}$ stage $(p \leq 0.001)$, bone metastasis $(p \leq 0.001)$, lung metastasis $(p \leq 0.001)$, brain metastasis $(p=0.007)$, chemotherapy $(p \leq 0.001)$ and local treatment $(p=0.002)$ were independent prognostic factors for $O S$, while gender $(p=0.078)$ and race $(p=0.126)$ were not (Table 2$)$. The indicators with $p$ value $<0.1$ were included in the multivariate cox proportional hazards regression analysis. The results revealed that age, gender, grade, histological type, bone metastasis, lung metastasis, brain metastasis, chemotherapy and local treatment were independent prognostic factors for OS. The corresponding survival curves are shown in Figure 2.

Table 2 Univariate and multivariate cox regression analyses for the presence of liver metastases at EC diagnosis 


\begin{tabular}{|c|c|c|c|c|}
\hline \multirow[t]{3}{*}{ Characteristic } & \multicolumn{2}{|l|}{ Univariate } & \multicolumn{2}{|l|}{ Multivariate } \\
\hline & Hazard ratio & $p$ value & Hazard ratio & $p$ value \\
\hline & $(95 \% \mathrm{Cl})$ & & $(95 \% \mathrm{Cl})$ & \\
\hline \multicolumn{5}{|l|}{ Gender } \\
\hline Male & Reference & 0.078 & Reference & \\
\hline Female & $0.887(0.769-1.022)$ & & $0.804(0.695-0.930)$ & 0.003 \\
\hline \multicolumn{5}{|l|}{ Race } \\
\hline White & Reference & 0.126 & - & - \\
\hline Black & $1.189(0.994-1.423)$ & & - & - \\
\hline Other & $1.038(0.827-1.302)$ & & - & - \\
\hline \multicolumn{5}{|l|}{ Age } \\
\hline$<57$ years & Reference & $\leq 0.001$ & Reference & \\
\hline $58-77$ years & $1.264(1.131-1.412)$ & & $1.076(0.961-1.205)$ & 0.204 \\
\hline$\geq 78$ years & $1.992(1.691-2.347)$ & & $1.388(1.172-1.645)$ & $\leq 0.001$ \\
\hline \multicolumn{5}{|l|}{ Pathological grade } \\
\hline Grades 1-2 & Reference & $\leq 0.001$ & Reference & \\
\hline Grades 3-4 & $1.201(1.086-1.329)$ & & $1.197(1.080-1.328)$ & 0.001 \\
\hline Histological type & & & & 0.006 \\
\hline Adenocarcinoma & Reference & $\leq 0.001$ & Reference & \\
\hline Squamous & $1.361(1.198-1.546)$ & & $1.241(1.079-1.426)$ & 0.002 \\
\hline Other & $1.162(0.883-1.529)$ & & $1.216(0.919-1.609)$ & 0.171 \\
\hline \multicolumn{5}{|l|}{ Primary tumor site } \\
\hline Lower & Reference & 0.002 & Reference & \\
\hline Upper+Middle & $1.232(1.067-1.421)$ & & $1.097(0.939-1.281)$ & 0.245 \\
\hline Overlapping lesion & $1.241(1.007-1.530)$ & & $1.192(0.964-1.474)$ & 0.105 \\
\hline \multicolumn{5}{|l|}{ T stage } \\
\hline Tx & Reference & 0.004 & Reference & \\
\hline $\mathrm{T} 1+\mathrm{T} 2$ & $0.889(0.787-1.005)$ & & $0.938(0.827-1.063)$ & 0.316 \\
\hline $\mathrm{T} 3+\mathrm{T} 4$ & $1.067(0.947-1.203)$ & & $1.061(0.940-1.198)$ & 0.337 \\
\hline
\end{tabular}




\begin{tabular}{|c|c|c|c|c|}
\hline No & Reference & $\leq 0.001$ & Reference & \\
\hline $\mathrm{N}+$ & $1.213(1.090-1.349)$ & & $1.051(0.939-1.176)$ & 0.403 \\
\hline \multicolumn{5}{|c|}{ Bone metastases } \\
\hline No & Reference & $\leq 0.001$ & Reference & \\
\hline Yes & $1.355(1.201-1.528)$ & & $1.310(1.157-1.483)$ & $\leq 0.001$ \\
\hline \multicolumn{5}{|c|}{ Lung metastases } \\
\hline No & Reference & $\leq 0.001$ & Reference & \\
\hline Yes & $1.291(1.162-1.435)$ & & $1.190(1.070-1.325)$ & 0.001 \\
\hline \multicolumn{5}{|c|}{ Brain metastases } \\
\hline No & Reference & 0.007 & Reference & \\
\hline Yes & $1.346(1.067-1.698)$ & & $1.369(1.079-1.738)$ & 0.010 \\
\hline \multicolumn{5}{|c|}{ Chemotherapy } \\
\hline No & Reference & $\leq 0.001$ & Reference & \\
\hline Yes & $0.252(0.227-0.281)$ & & $0.267(0.239-0.298)$ & $\leq 0.001$ \\
\hline \multicolumn{5}{|c|}{ Local treatment } \\
\hline No & Reference & 0.002 & Reference & \\
\hline Yes & $0.862(0.778-0.954)$ & & $0.855(0.769-0.951)$ & 0.004 \\
\hline
\end{tabular}

Abbreviation: EC, esophageal cancer, $\mathrm{T}$, tumor, $\mathrm{N}$, lymph node, local treatment, surgery or radiation, $\mathrm{Cl}$, confidence interval.

\section{Nomogram}

A visual nomogram was constructed to predict 6-month and 1-year OS based on the multivariate cox proportional hazard regression analysis. Each variable was given a score on a points scale (Figure 3). The sum of these numbers was located on the total points axis. By summing the total scores projected on the bottom scale, we estimated the probability of 6-month and 1-year OS. In our newly visualized nomogram, based on internal validation, the corrected C-index for predicted 6-month OS and 1-year OS was 0.735 (95\% Cl: $0.723-0.748)$. The calibration plot for the probability of 6-month and 1-year OS showed good correlation between the nomogram prediction and actual observation (Figure 4). DCA was employed to further verify the clinical validity of the model, and it demonstrated good clinical applicability of the nomogram (Figure 5). 


\section{Discussion}

EC patients with metastatic diseases differ from patients without metastasis in epidemiology, biology, and prognosis. Approximately $50 \%$ of patients present with metastases to distant lymph nodes or organs at initial diagnosis ${ }^{[4]}$. The prognosis of metastatic EC is poor, and the five-year survival rate is less than $5 \%{ }^{[7,9]}$. We conducted an updated population-based study to investigate the epidemiological characteristics for ECLM. Moreover, a visual nomogram was established to predict individualized survival and to make treatment recommendations for patients with ECLM.

Previous epidemiological studies on ECLM have shown that the prognosis is poor ${ }^{[9,11]}$. Haiqi He et al reported that the age-adjusted incidence of EC increased from 5.55 to 7.44 per 100,000 person-years between 1973 and 2004. Later, the rate decreased at an annual percentage change of $1.23 \%{ }^{[2]}$. Studies have shown that the liver is one of the most common sites of metastases, occurring in $35-40 \%$ of patients at the time of EC diagnosis ${ }^{[6,14]}$, and it is the first site of recurrence in $6-25 \%$ of patients after esophagectomy ${ }^{[15]}$. Similar retrospective studies of patients with DM have reported that the most common sites of DM are the liver, followed by the distant lymph nodes, lung, bone, and brain ${ }^{[15,16]}$. In the present study, the incidence of ECLM increased with age (Figure 1A), but survival rates showed the opposite trend (Figure 1D). We analyzed the annual incidence of patients in different age groups from 2010 to 2016. As age increased, the incidence increased annually, peaking at age 70 to 79 years and then decreasing. The highest incidence of metastasis was in the liver, followed by the lung, bone and brain. All patients with metastases at the four most common sites had a poorer prognosis than those without metastasis, and patients with bone metastasis of EC had the worst prognosis. Moreover, liver metastases were associated with better OS than bone metastases.

There are limited studies on the effect of the site of DM on survival in patients with metastatic EC. Chen et al found that DM was not associated with OS in patients with metastatic squamous cell esophageal cancer ${ }^{[16]}$. Tanaka et al also observed no significant difference in the median survival time based on the site of DM (liver, bone, and lung, $p=0.8786$ ) ${ }^{[7]}$. Blank et al found that the localization and number of metastases were not significant prognostic factors for survival ${ }^{[17]}$. However, they did not further analyze the effect of site-specific DM on survival. San-gang Wu et al reported that bone metastases were associated with poorer OS than liver metastases, especially in patients with esophageal $A C{ }^{[4]}$. They also observed similar OS rates for esophageal SCC patients with liver, bone, or lung metastases. As shown in Figure 1C, all patients with metastases at the four most common sites had a worse prognosis than those without metastasis, with EC patients with bone metastasis having the worst outcome (Figure 1C). The four survival curves demonstrated similar OS rates for EC patients with liver, bone, brain or lung metastasis. We further screened that liver metastases, bone metastasis, lung metastasis, and brain metastasis were independent prognostic factors for OS. Therefore, the classification of patients with metastatic EC by site-specific metastasis may help physicians more accurately assess prognosis and generate treatment strategies. 
We chose a series of significant factors to generate a nomogram. In our study, we found that gender was an independent predictive factor for liver metastasis in patients with EC. The incidence of liver metastasis was $86.2 \%(1631 / 1892)$ in male patients and 13.8\% (261/1892) in female patients. A previous study demonstrated that the influence of sex hormones may explain the sex difference in EC incidence ${ }^{[18]}$. Our results also indicate that EAC is the most prevalent type of EC in the U.S. EAC patients accounted for $80 \%$ of the patient population and had a worse prognosis than squamous cell cancer patients. A related study reported that the incidence of EAC has increased since 1973 and surpassed that of squamous cell cancer ${ }^{[2]}$. The sharp increase in EAC incidence in the U.S. can be partly explained by the increasing numbers of overweight individuals or individuals with gastroesophageal reflux disease (GERD), which is associated with obesity ${ }^{[19]}$.

Previous studies have demonstrated that $\mathrm{T}$ stage and $\mathrm{N}$ stage are predictors for the DM of EC. Zhang et al showed that T stage (T3-4 vs. T1-2) and N stage (N1 vs. N0) were important prognostic factors for metastasis-free survival ${ }^{[20]}$. Sakanaka et al. revealed that metastatic lymph nodes were related to the occurrence of DM, and patients with larger metastatic lymph nodes had a higher risk of DM ${ }^{[21]}$. In our univariate analysis, T stage $(p=0.004)$ and $\mathrm{N}$ stage $(p \leq 0.001)$ were significant prognostic factors for ECLM. In contrast to a previous study, the multivariate logistic regression analysis showed that $\mathrm{T}$ stage $(p=0.316)$ and $\mathrm{N}$ stage $(p=0.403)$ were not significant indicators for survival.

Chemotherapy is the standard treatment for metastatic EC. With the current chemotherapy regimen, the duration of the response is short, and survival is usually 6 to 8 months ${ }^{[22]}$. Compared to the survival rate of patients who receive best supportive care, the survival rate of patients who receive chemotherapy alone is significantly higher $(p=0.061)^{[16]}$. Chemotherapy increases the number of patients who are able to undergo resection or radiotherapy ${ }^{[23]}$. However, there is no consensus on whether palliative radiotherapy or surgery is of value in metastatic EC. Several studies have suggested that palliative radiotherapy and surgery can improve survival in patients with metastatic EC $[9,11,24]$. Moreover, several retrospective studies have found that primary tumor resection may be considered a strategy for selected patients with stage IV EC who achieve a favorable response to systemic chemotherapy ${ }^{[17,25]}$. Another study of the SEER database also found that surgery and preoperative radiotherapy were associated with improved survival in patients with metastatic EC ${ }^{[9]}$. In the present study, most patients received chemotherapy, 39 (1.60\%) patients received primary surgery, and 632 (33.40\%) patients received radiotherapy. The survival analyses suggested that local treatment and chemotherapy improve survival in ECLM patients. Local treatment $(p=0.004)$ and chemotherapy $(p \leq 0.001)$ were identified as independent favorable prognostic factors for OS, similar to results in metastatic breast cancer and colorectal cancer ${ }^{[26,27]}$. However, the number of patients who were suitable for local treatment of the primary tumor was not enough to reach definite conclusions. Therefore, further studies are required to explore the subgroups of patients who may benefit from local therapy.

The prognostic study of EC will be useful for clinical practice. Therefore, there are a number of nomograms for metastatic EC. For example, Chao Zhu et al. developed a nomogram to evaluate the risk 
factors for DM in patients with EC, and the prediction model presented good discrimination, with an AUC of 0.738 and a C-index of 0.747 (95\% confidence interval: $0.734-0.760)^{[28]}$. Other researchers have specialized in evaluating the clinicopathologic characteristics of long-term survival in patients with metastatic EC. For example, Mina Liu et al. developed a nomogram for EC [29], and the OS nomogram had a C-index of 0.633 . However, previous studies did not focus on less common sites of metastasis. Therefore, we conducted the present study to focus on liver metastasis. For this reason, we included a scope of significant variables to achieve the widest applicability. The prediction model presented good discrimination, with a C-index of 0.735 (95\% Cl: $0.723-0.748)$, and the calibration plot showed satisfactory calibration. The nomogram also demonstrated good clinical applicability.

Our study also has some shortcomings, such as the lack of some key details, especially the use of chemotherapy or radiotherapy, and surgery details are not described in the SEER database. Second, because of a lack of data support from another database, we did not perform an external validation. Therefore, the presented nomogram needs to be replicated and then prospectively validated before it can be implemented in clinical practice.

\section{Conclusions}

This study provides new insights into the epidemiology of patients with ECLM. Age, gender, tumor characteristics, and therapy should be considered when predicting the prognosis of patients with ECLM. The nomogram described herein may improve clinicians' abilities to predict individualized survival and make treatment recommendations for patients with ECLM.

\section{Abbreviations}

DM: distant metastasis

ECLM: esophageal cancer with liver metastasis

SEER: Surveillance, Epidemiology, and End Results database

EC: esophageal cancer

EAC: esophageal adenocarcinoma

ESC: esophageal squamous carcinoma

OS: overall survival

C-index: concordance index

DCA: decision curve analysis

T stage: tumor stage 
N stage: lymph node stage

\section{Declarations}

Acknowledgements

We are thankful for the contribution of the SEER database and all authors.

Authors' contributions

(I) Conception and design: Zhenfei Xiang and Huacai Xiong conceived and designed the study, (II) Acquisition of data: Wei Jiang was responsible for this work, (III) Data analysis and interpretation: Mingyao Li and Tian Chen performed the statistical analysis, (IV) Manuscript writing: Danfei Hu and Huacai Xiong analysed and interpreted the data, drafted the manuscript and revised it critically for important content, (V) Final approval of manuscript: All authors.

Competing of interests

The authors declare that they have no competing interests.

Availability of data and materials

All data generated or analysed during this study are included in this published article and its supplementary information files.

Ethics approval and consent to participate

This study was approved by Ningbo Medical Center Lihuili Hospital ethics committee. Analysis of the raw data from the SEER program was exempt from medical ethics review, and no informed consent was required.

Consent for publication

All authors approve for publication.

Funding statement

The present study was supported by the Natural Science Foundation of Ningbo (2021J289) and Ningbo Clinical Research Center for thoracic \& breast neoplasms (2021L002).

\section{References}

1. COLLABORATORS. G O C. The global, regional, and national burden of oesophageal cancer and its attributable risk factors in 195 countries and territories, 1990-2017: a systematic analysis for the 
Global Burden of Disease Study 2017 [J]. The lancet Gastroenterology \& hepatology, 2020, 5(6):582597.

2. $\mathrm{HE} \mathrm{H}, \mathrm{CHEN} \mathrm{N}, \mathrm{HOU} Y$, et al. Trends in the incidence and survival of patients with esophageal cancer: A SEER database analysis [J]. Thorac Cancer, 2020, 11(5): 1121-1128.

3. XIE S H, LAGERGREN J. Time trends in the incidence of oesophageal cancer in Asia: Variations across populations and histological types [J]. Cancer Epidemiol, 2016, 44:71-76.

4. WU S G, ZHANG W W, HE Z Y, et al. Sites of metastasis and overall survival in esophageal cancer: a population-based study [J]. Cancer Manag Res, 2017, 6(9):781-788.

5. WU S-G, XIE W-H, ZHANG Z-Q, et al. Surgery Combined with Radiotherapy Improved Survival in Metastatic Esophageal Cancer in a Surveillance Epidemiology and End Results Population-based Study [J]. Scientific Reports, 2016, 6(1): 28280.

6. BRAY F, FERLAY J, SOERJOMATARAM I, et al. Global cancer statistics 2018: GLOBOCAN estimates of incidence and mortality worldwide for 36 cancers in 185 countries [J]. CA Cancer J Clin, 2018, 68(6): 394-424.

7. TANAKA T, FUJITA H, MATONO S, et al. Outcomes of multimodality therapy for stage IVB esophageal cancer with distant organ metastasis (M1-Org) [J]. Dis Esophagus, 2010, 23(8): 646-651.

8. WANG J, SURI J S, ALLEN P K, et al. Factors Predictive of Improved Outcomes With Multimodality Local Therapy After Palliative Chemotherapy for Stage IV Esophageal Cancer [J]. Am J Clin Oncol, 2016, 39(3): 228-235.

9. WU S G, XIE W H, ZHANG Z Q, et al. Surgery Combined with Radiotherapy Improved Survival in Metastatic Esophageal Cancer in a Surveillance Epidemiology and End Results Population-based Study [J]. Sci Rep, 2016, 6(1):28280.

10. GUTTMANN D M, MITRA N, BEKELMAN J, et al. Improved Overall Survival with Aggressive Primary Tumor Radiotherapy for Patients with Metastatic Esophageal Cancer [J]. J Thorac Oncol, 2017, 12(7): 1131-1142.

11. HUDDY J R, THOMAS R L, WORTHINGTON T R, et al. Liver metastases from esophageal carcinoma: is there a role for surgical resection? [J]. Dis Esophagus, 2015, 28(5): 483-487.

12. HUANG Y X, LIN Y Z, LI J L, et al. Role of postoperative radiotherapy in pT3NO rectal cancer: A riskstratification system based on population analyses [J]. Cancer Med, 2019, 8(3): 1024-1033.

13. ROBERT L. CAMP, MARISA DOLLED-FILHART, RIMM D L. X-tile『A New Bio-Informatics Tool for Biomarker Assessment and Outcome-Based Cut-Point Optimization [J]. Clin Cancer Res, 2004, (10): 7252-7259.

14. ARNOLD M, SOERJOMATARAM I, FERLAY J, et al. Global incidence of oesophageal cancer by histological subtype in 2012 [J]. Gut, 2015, 64(3): 381-387.

15. MARIETTE C, BALON J M, PIESSEN G, et al. Pattern of recurrence following complete resection of esophageal carcinoma and factors predictive of recurrent disease [J]. Cancer, 2003, 97(7): 16161623. 
16. CHEN M Q, XU B H, ZHANG Y Y. Analysis of prognostic factors for esophageal squamous cell carcinoma with distant organ metastasis at initial diagnosis [J]. J Chin Med Assoc, 2014, 77(11): 562-566.

17. BLANK S, LORDICK F, DOBRITZ M, et al. A reliable risk score for stage IV esophagogastric cancer [J]. European Journal of Surgical Oncology (EJSO), 2013, 39(8): 823-830.

18. MATHIEU L N, KANAREK N F, TSAI H L, et al. Age and sex differences in the incidence of esophageal adenocarcinoma: results from the Surveillance, Epidemiology, and End Results (SEER) Registry (1973-2008) [J]. Dis Esophagus, 2014, 27(8): 757-763.

19. RILEY C A, WU E L, HSIEH M C, et al. Association of Gastroesophageal Reflux With Malignancy of the Upper Aerodigestive Tract in Elderly Patients [J]. JAMA Otolaryngol Head Neck Surg, 2018, 144(2): 140-148.

20. ZHANG D, ZHOU X, BAO W, et al. Plasma fibrinogen levels are correlated with postoperative distant metastasis and prognosis in esophageal squamous cell carcinoma [J]. Oncotarget, 2015, 6(35): 38410-38420.

21. SAKANAKA K, ISHIDA Y, ITASAKA S, et al. Identification of a predictive factor for distant metastasis in esophageal squamous cell carcinoma after definitive chemoradiotherapy [J]. Int J Clin Oncol, 2016, 21(5): 899-908.

22. PROCOPIO F, MARANO S, GENTILE D, et al. Management of Liver Oligometastatic Esophageal Cancer: Overview and Critical Analysis of the Different Loco-Regional Treatments [J]. Cancers, 2019, 12(1): 20.

23. ANDREOU A, VIGANò L, ZIMMITTI G, et al. Response to preoperative chemotherapy predicts survival in patients undergoing hepatectomy for liver metastases from gastric and esophageal cancer [J]. Journal of gastrointestinal surgery : official journal of the Society for Surgery of the Alimentary Tract, 2014, 18(11): 1974-1986.

24. XU J, LU D, ZHANG L, et al. Palliative resection or radiation of primary tumor prolonged survival for metastatic esophageal cancer [J]. Cancer Med, 2019, 8(17): 7253-7264.

25. WANG J, SURI J S, ALLEN P K, et al. Factors Predictive of Improved Outcomes With Multimodality Local Therapy After Palliative Chemotherapy for Stage IV Esophageal Cancer [J]. American journal of clinical oncology, 2016, 39(3): 228-235.

26. WU S G, ZHANG W W, SUN J Y, et al. The survival benefits of local surgery in stage IV breast cancer are not affected by breast cancer subtypes: a population-based analysis [J]. Oncotarget, 2017, 8(40): 67851-67860.

27. ZHANG X L, LIU R F, ZHANG D, et al. Laparoscopic versus open liver resection for colorectal liver metastases: A systematic review and meta-analysis of studies with propensity score-based analysis [J]. International journal of surgery (London, England), 2017, 44:191-203.

28. ZHU C, YOU Y, LIU S, et al. A Nomogram to Predict Distant Metastasis for Patients with Esophageal Cancer [J]. Oncol Res Treat, 2020, 43(1-2): 2-9. 
29. LIU M, WANG C, GAO L, et al. A nomogram to predict long-time survival for patients with $M 1$ diseases of esophageal cancer [J]. J Cancer, 2018, 9(21): 3986-3990.

\section{Figures}

A

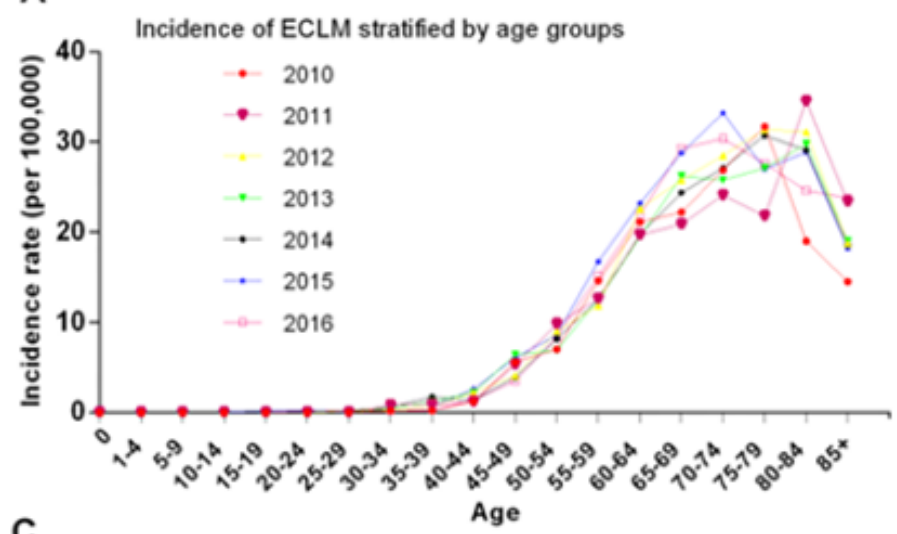

C

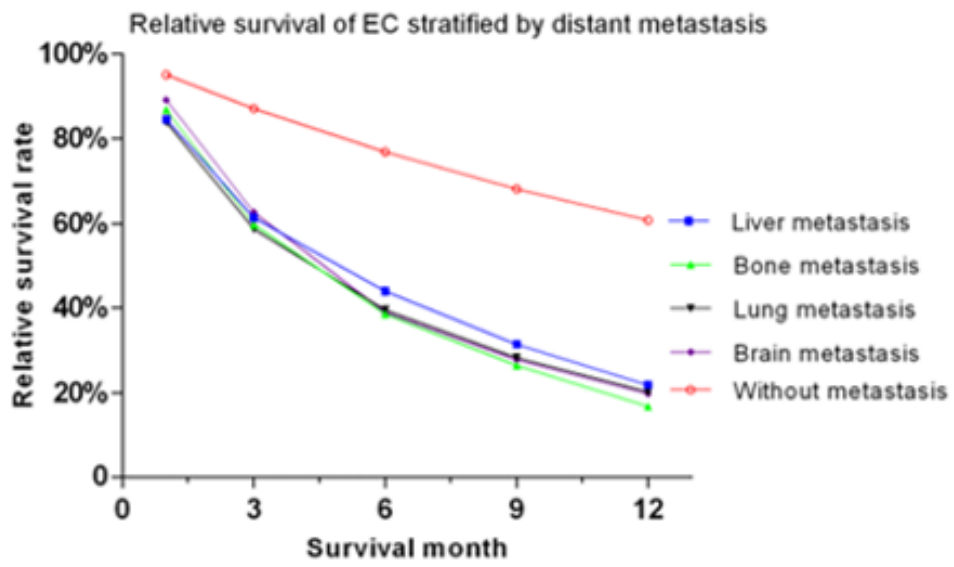

B
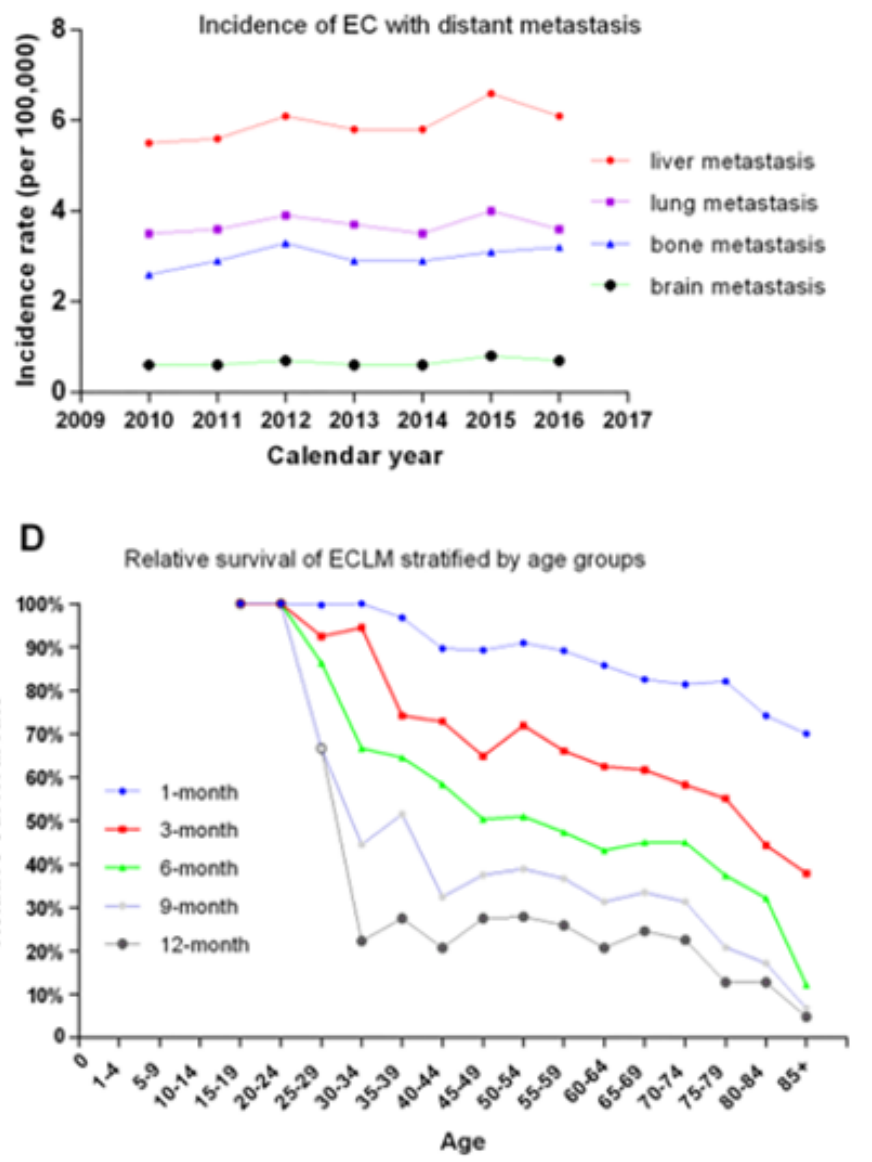

\section{Figure 1}

(A) Incidence of esophageal cancer with liver metastasis (ECLM) stratified by age group; (B) incidence of ECLM; (C) relative survival of esophageal cancer patients stratified by sites of $D M$; (D) relative survival of ECLM patients stratified by age group. 
A
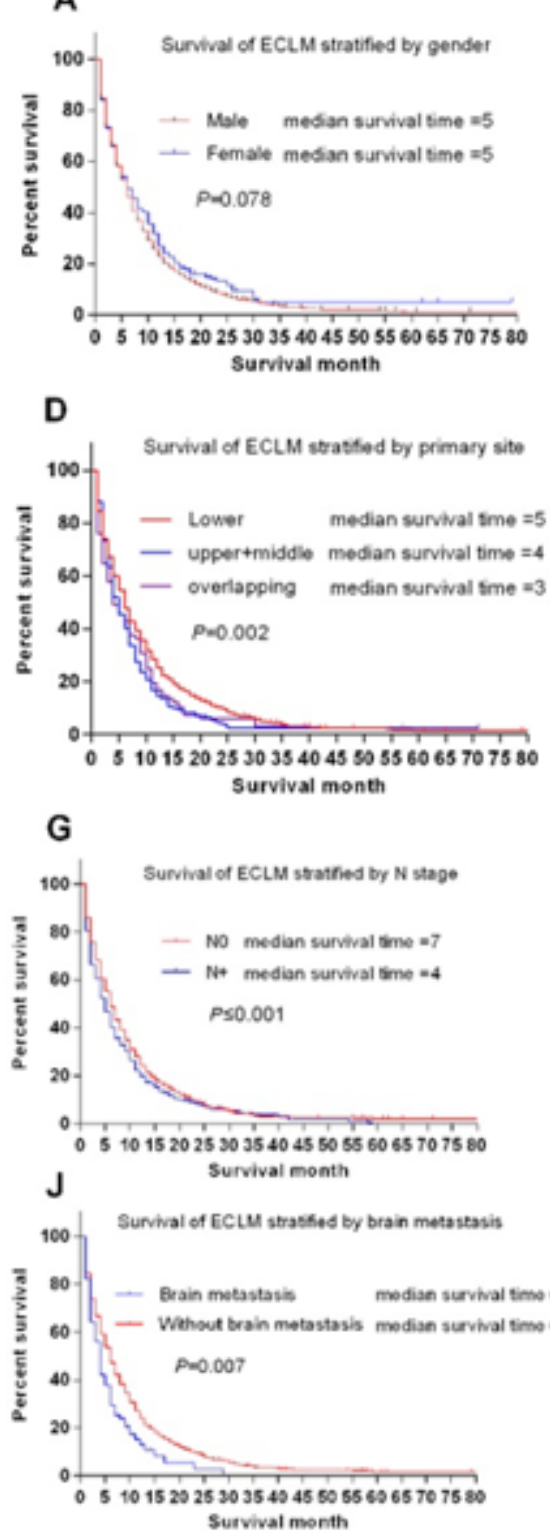

B

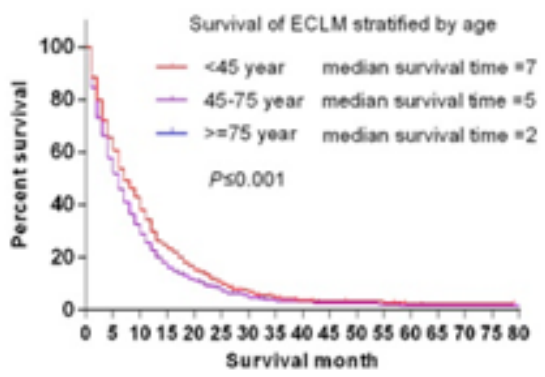

\section{E}

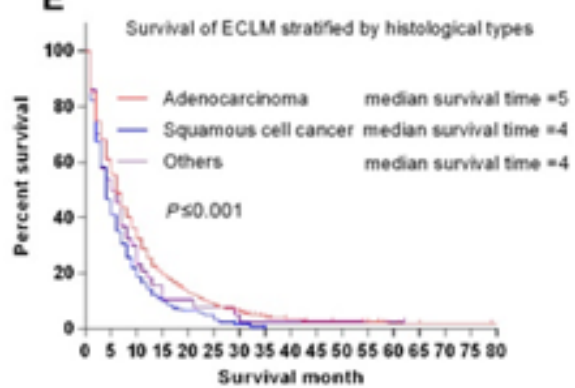

H

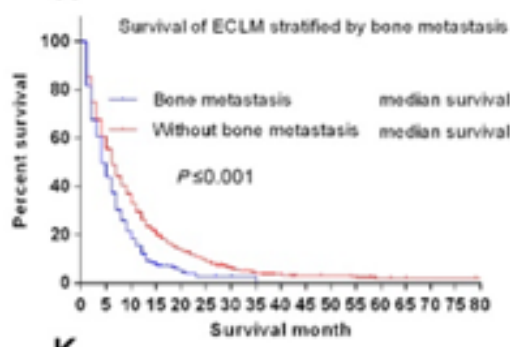

$\mathrm{K}$

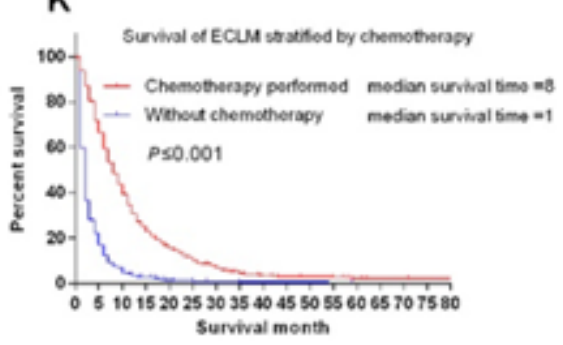

C

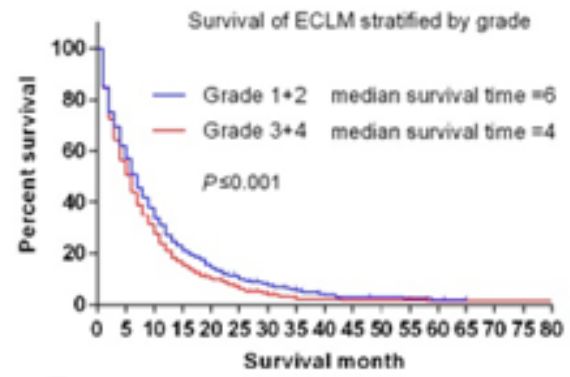

F

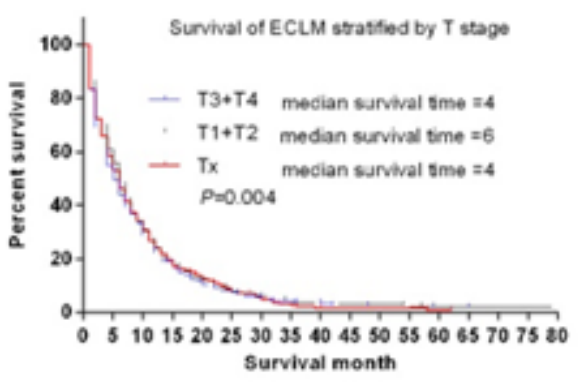

I

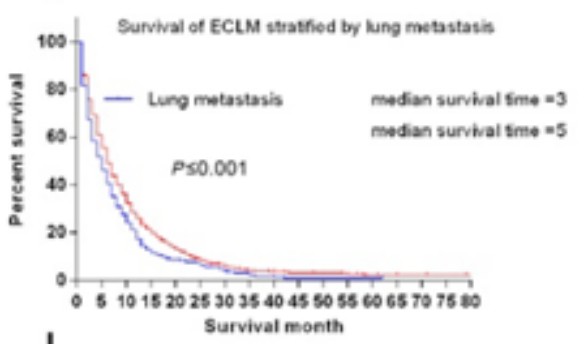

L

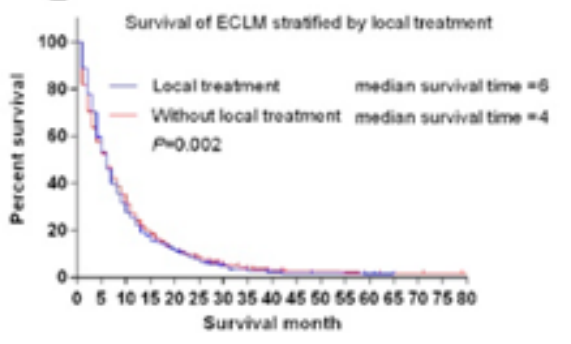

Figure 2

(A) Survival of ECLM patients stratified by gender; (B) survival of ECLM patients stratified by age; (C) survival of ECLM patients stratified by grade; (D) survival of ECLM patients stratified by primary tumor site; (E) survival of ECLM patients stratified by histological type; (F) survival of ECLM patients stratified by T stage; $(\mathrm{G})$ survival of ECLM patients stratified by $\mathrm{N}$ stage; $(\mathrm{H})$ survival of ECLM patients stratified by bone metastasis; (I) survival of ECLM patients stratified by lung metastasis; (J) survival of ECLM patients stratified by brain metastasis; (K) survival of ECLM patients stratified by chemotherapy; (L) survival of ECLM patients stratified by local treatment. 


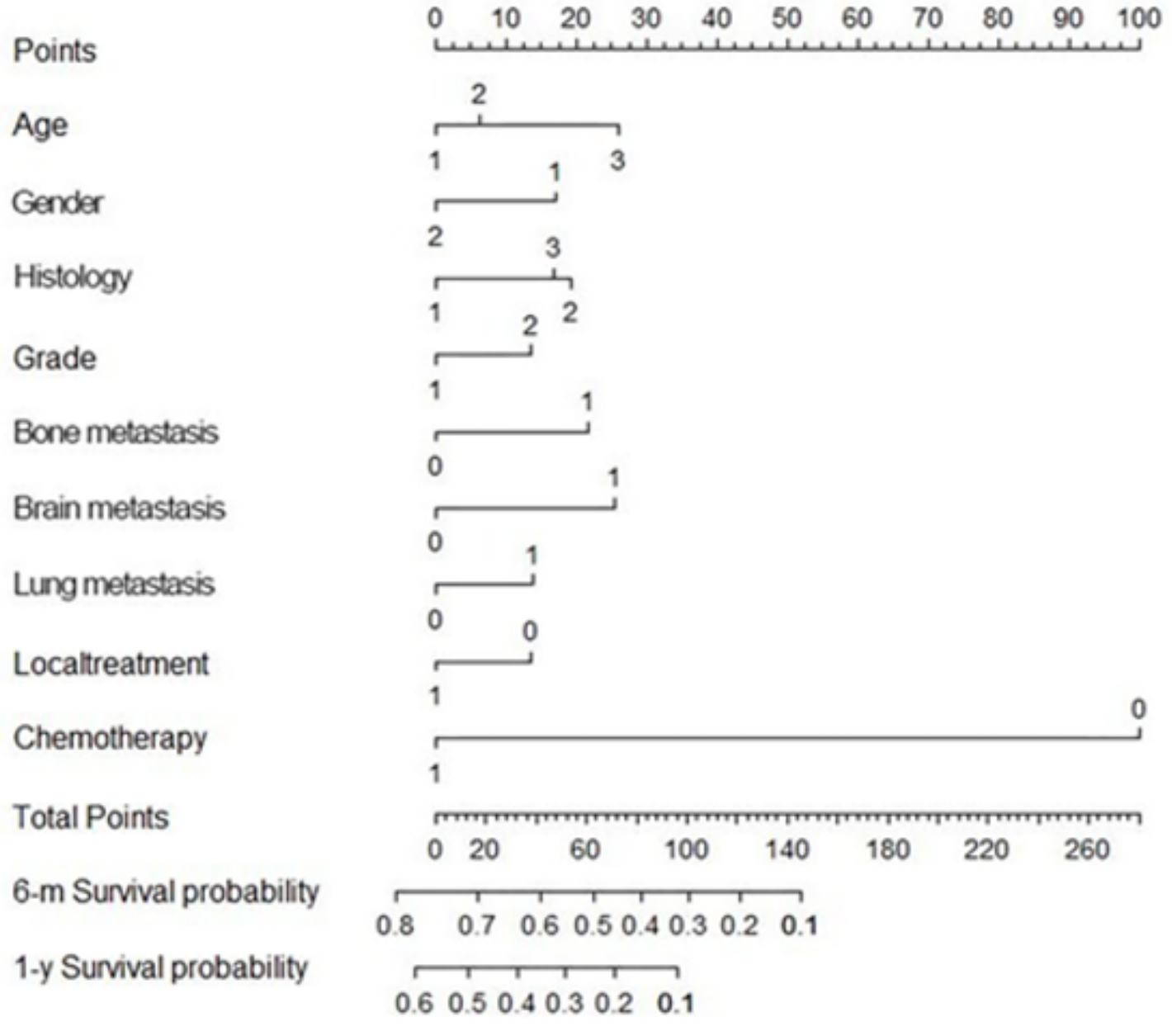

\section{Figure 3}

Overall survival nomogram for ECLM patients. 


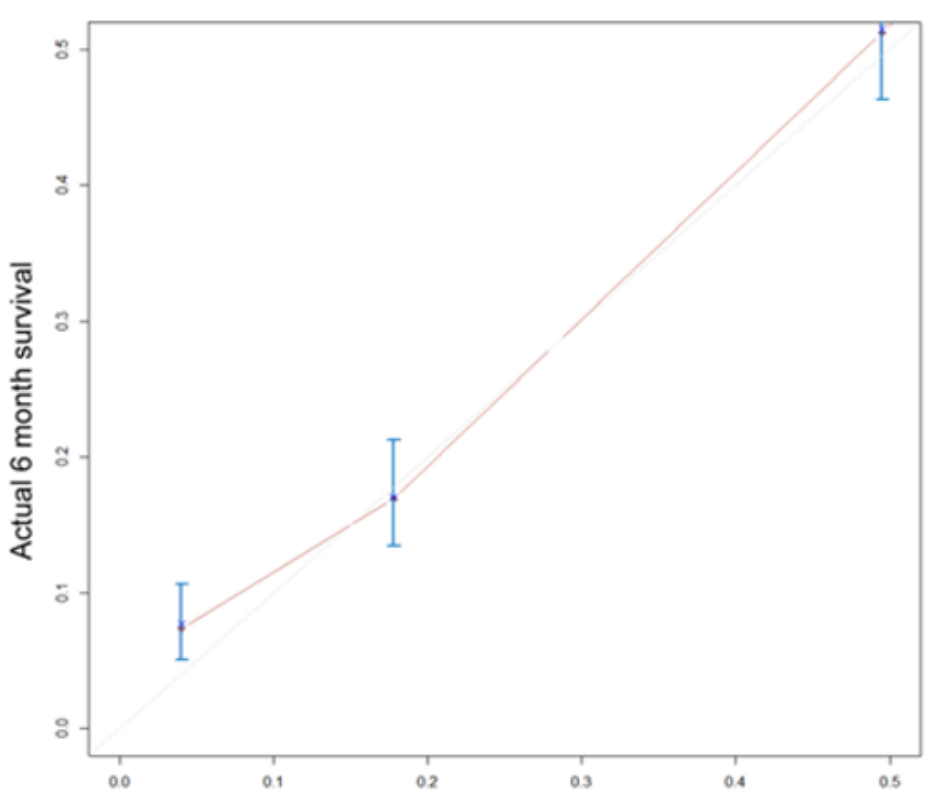

A Nomogram predicted 6 month survival

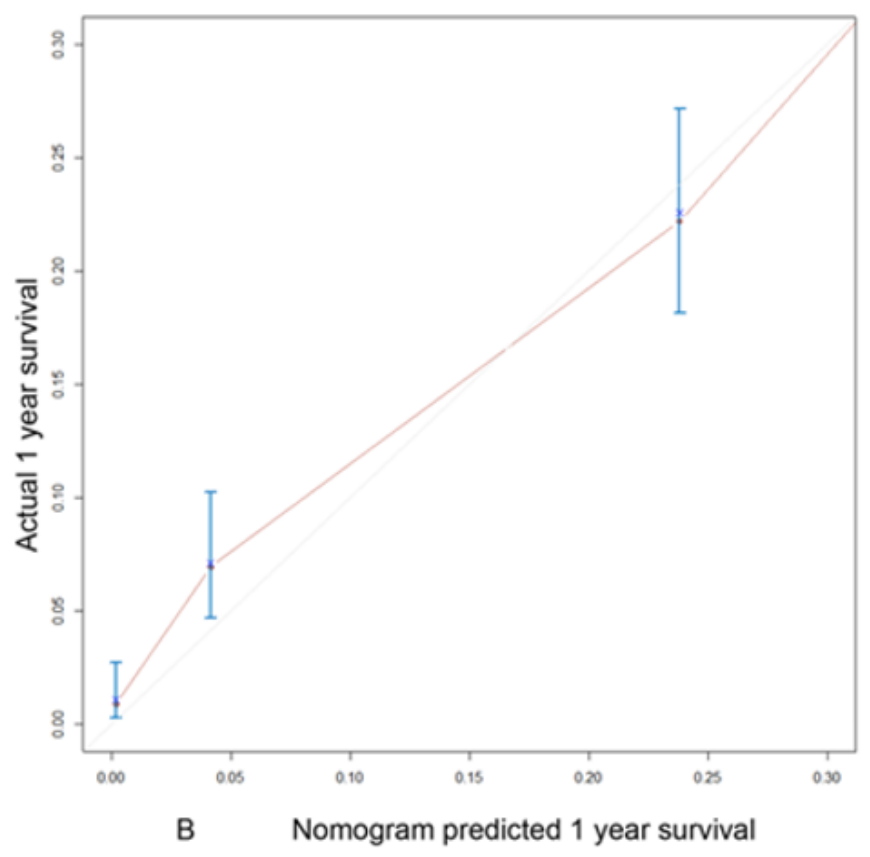

Figure 4

Calibration plot showing nomogram-predicted 6-month and 1-year OS ECLM probabilities.

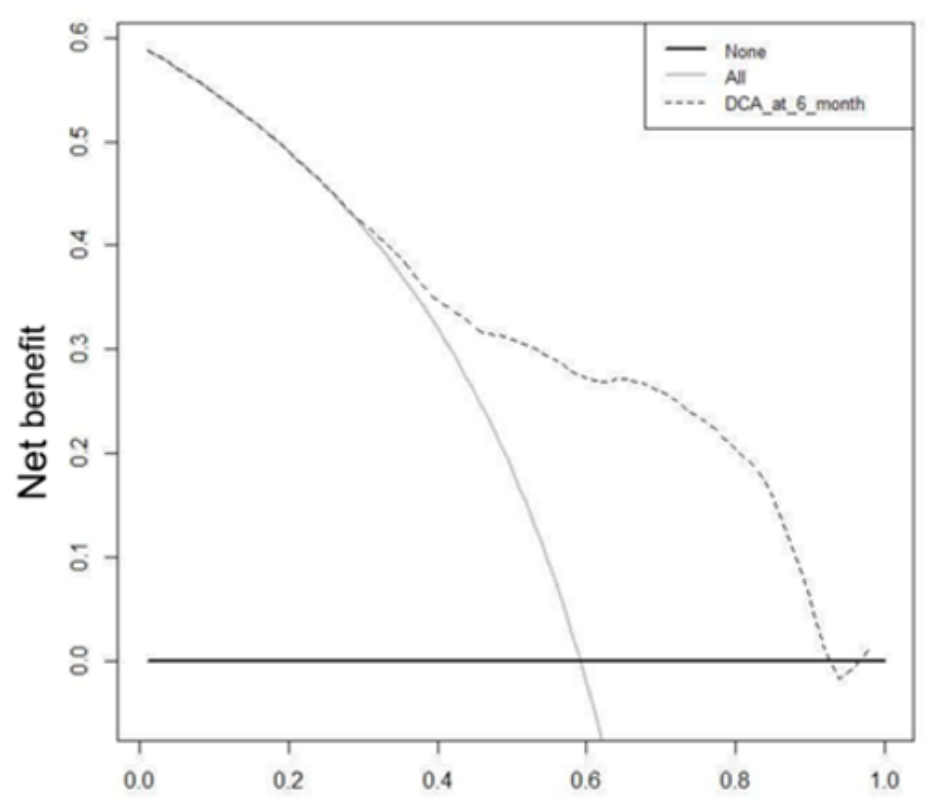

A Treshhold probability

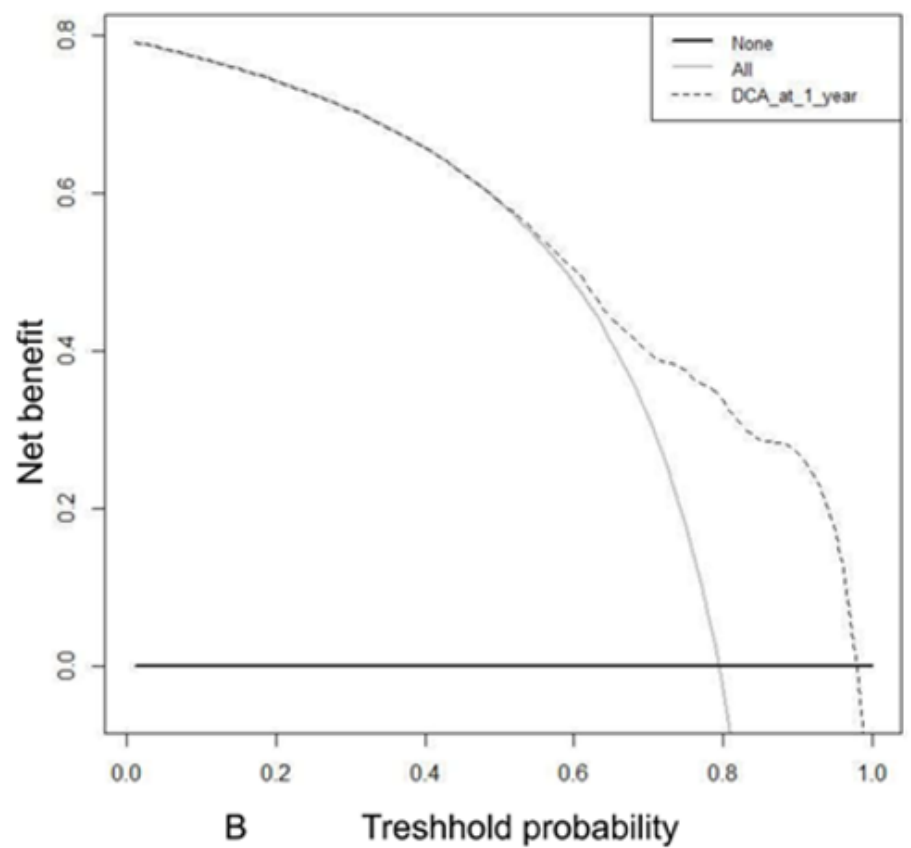

B Treshhold probability

\section{Figure 5}

Decision curve analysis (DCA) for the nomogram and conventional forecasting indicators, including age, gender, histological type, grade, bone metastasis, lung metastasis, brain metastasis, chemotherapy and 
local treatment.

\section{Supplementary Files}

This is a list of supplementary files associated with this preprint. Click to download.

- Rawdata.slm

- Rawdata.xlsx 